-1400012081
cepiat

The design of a parallel algorithm to solve the word problem for the free partially commutative groups

Joaquim Gabarró

Report LSI-91-39

FACULTAT D'IFFGRMȦIICA BIBLIOTECA

R. $9.173 \quad 15$ OCT. 1991 


\title{
The design of a parallel algorithm to solve the word problem for the free partially commutative groups
}

\author{
J. Gabarró * \\ Dept. de Llenguatges i Sistemes Informàtics \\ Universitat Politècnica de Catalunya \\ Pau Gargallo 5 , 08028 Barcelona \\ Spain \\ Schlo $\beta$ Dagstuhl / Draft / October 101991
}

\begin{abstract}
We develop a parallel algorithm to solve the word problem for free partially commutative groups. These groups were introduced by $\mathrm{C}$. Wrathall to generalize free groups. We represent the elements of these groups as a certain type of acyclic labeled graphs called dependency graphs. These graphs were introduced by A.Mazurkiewicz to model concurrent systems.

- First we study the parallel complexity of some basic problems arising in the study of dependency graphs. Such as correctness, isomorphism and relations with traces. Parallel algorithms are developed to solve all of them.

- Second we consider the combinatorial properties of free partially commutative groups. To do this we associate to every group a rewriting system over dependency graphs. Finally we apply all these ideas to solve in parallel the word problem.
\end{abstract}

Special emphasis is given in the design of the algorithms. The modular approach is widely used to obtain readable programs. It seems that many of the structuring techniques developed in sequential programming can be used directly in the PRAM context.

Keywords: PRAMS, data parallel algorithms, modular design, trace theory, free groups, free partially commutative groups, word problems, parallel complexity classes, $N C^{*}, N L^{*}, A C^{0}$, complete problems.

* Research supported by the ESPRIT II Basic Research Actions Program of the EC under contract No. 3075 (project ALCOM). 


\section{The parallel complexity of some dependency graphs problems.}

We would like to develop a parallel algorithm to solve word problem for the free partially commutative groups. To do this we need:

- Basic facts on complexity classes in the line of [BDG88] and [BDG90]. The fundamental paper on parallel complexity classes is [Co85]. In [ABGS91] we present how to reason, in terms of complexity theory, about sequential and parallel programs.

- Elements of partially commutative monoids [Ma88] and free partially commutative groups [Wr88].

- How to write data parallel programs and reason about them. Examples of data parallel algorithms are in [HS86]. In [GG91] it is introduced an axiomatic way to reason about these programs. It seems that the design of data parallel and sequential algorithms is very close.

Let us recall some basic concepts on traces and dependency graphs. A basic reference is [Ma88]. Given a finite set of events $\Sigma$ and a symmetric and irreflexive relation $\theta \subseteq \Sigma \times \Sigma$ we call the pair $(\Sigma, \theta)$ concurrent alphabet. The relation $\bar{\theta}=\Sigma \times \Sigma \backslash \theta$ is called conflict relation. The elements of the quotient monoid $\Sigma^{*} / \theta$ are called traces. Sometimes is better to represent traces by graphs, which makes explicit the ordering of symbol occurrences within the traces.

Dependency graphs. A triple $\gamma=(V, R, \varphi)$ is a dependency graph over $(\Sigma, \theta)$ when $V$ is a finite set called the nodes of $\gamma, R \subseteq V \times V$ is the set of arcs and $\varphi: V \rightarrow \Sigma$ is the labelling, if the following conditions holds:

- Acyclicity written as $R^{+} \cap\{(v, v) \mid v \in V\}=\emptyset$.

- Dependence connectivity given by

$$
R \cup R^{-1} \cup\{(v, v) \mid v \in V\}=\left\{\left(v, v^{\prime}\right) \in V \times V \mid\left(\varphi(v), \varphi\left(v^{\prime}\right)\right) \in \bar{\theta}\right\}
$$

The number of nodes of $\gamma$ written as $\#(\gamma)$ is $\#(V)$. We write $\Gamma(\Sigma, \theta)$ for the set of dependency graphs. Two dependency graphs $\gamma$ and $\delta$ are isomorphic, $\gamma \simeq \delta$, when there exists a bijection of nodes preserving labelling and arc connections. Given a word $w=$ $x_{1} \ldots x_{n} \in \Sigma^{*}$ we define the dependency graph associated to $w$ as $d(w)=\left(V_{w}, R_{w}, \varphi_{w}\right)$ where $V_{w}=\{1, \ldots, n\}, \varphi_{w}(i)=x_{i}$ and $R_{w}=\left\{(i, j) \mid(i<j) \wedge\left(x_{i}, x_{j}\right) \in \bar{\theta}\right\}$. A linearization of $\gamma$ is a word $w$ such that $\gamma \simeq d(w)$.

Composition. Given $\gamma=\left(V_{1}, R_{1}, \varphi_{1}\right)$ and $\delta=\left(V_{2}, R_{2}, \varphi_{2}\right)$ with $V_{1} \cap V_{2}=\emptyset$, the composition $\gamma \circ \delta=(V, R, \varphi)$ is defined as:

- $V=V_{1} \cup V_{2}$.

- $R=R_{1} \cup R_{2} \cup\left\{\left(v_{1}, v_{2}\right) \in V_{1} \times V_{2} \mid\left(\varphi_{1}\left(v_{1}\right), \varphi_{2}\left(v_{2}\right)\right) \in \bar{\theta}\right\}$.

- $\varphi=\varphi_{1} \cup \varphi_{2}$.

Denoting the empty graph as $\lambda=(\emptyset, \emptyset, \emptyset)$, we have that $(\Gamma(\Sigma, \theta), \circ, \lambda)$ is a monoid. Identifying dependency graphs with classes in $\Sigma^{*} / \theta$ we get an isomorphism between $\Sigma^{*} / \theta$ and $\Gamma(\Sigma, \theta)$. 
Hiding. Let us consider how to hide some events $V \subseteq V^{\prime}$ in a dependency graph $\gamma=$ $\left(V^{\prime}, R, \varphi\right)$. The result is written as $\gamma \backslash V=(V, R \cap V \times V, \varphi \mid V)$. Given a graph and a vertex $v$ of this graph the set of ancestors and descendants [Ja88] are:

$$
<v>^{*}=\left\{v^{\prime} \mid v^{\prime} \stackrel{*}{\longrightarrow} v\right\} \quad \Delta^{*}(v)=\left\{v^{\prime} \mid v \stackrel{*}{\longrightarrow} v^{\prime}\right\}
$$

We adapt these ideas to deal with a dependency graph $\delta=(V, R, \varphi)$ defining, for $v \in V$ :

- The dependency graph $\langle v, \delta\rangle^{*}=\delta \backslash \overline{\langle v\rangle^{*}}$ determined by taking all the ancestors of $v$ in $\gamma$.

- The dependency graph $\Delta^{*}(v, \delta)=\delta \backslash \overline{\Delta^{*}(v)}$ obtained by taking all the descendants of $v$ in $\gamma$.

The figure 1 give us examples of basic operations in $\Gamma(\Sigma, \theta)$.

Problem 1: The dependency graphs problem for concurrent alphabets, called $D E P E N$ DENCY _ GRAPHS C CORRECTNESS is the following:

Input: A concurrent alphabet $(\Sigma, \theta)$ and $\gamma=(V, R, \varphi)$.

Output: It is true that $\gamma \in \Gamma(\Sigma, \theta)$ ?.

Lemma 2: The problem DEPENDENCY_GRAPHS_CORRECTNESS is $N L^{*}$ complete.

Proof. First let us prove that:

DEPENDENCY_GRAPH_CORRECTNESS $<_{N C^{1}}$ TRANSITIVE_CLOSURE.

We represent the circuit given the $N C^{1}$ reduction as a PRAM program (figure 2). This program can be easily unfolded to give us a circuit.

Let us prove completeness. Let $M$ be a log space Turing machine working in time $n^{k}$. We assume that $M$ has a clock, in such a way that every configuration has a well defined time $t$, we write $c(t)$. The initial configuration is $c_{i}(0)$ and the unique final configuration is $c_{f}\left(n^{k}\right)$. The only possible transitions are from time $t$ to time $t+1$.

Let us reduce this problem to DEPENDENCY_GRAPH_CORRECTNESS using $N C^{1}$ reductions. Given $\left\langle M, c_{i}(0), c_{f}\left(n^{k}\right)\right\rangle$ let us construct $\left\langle\left(\Sigma_{M}, \theta_{M}\right), \gamma_{M}\right\rangle$ such that

$$
c_{i}(0) \stackrel{*}{\vdash} c_{f}\left(n^{k}\right) \Longleftrightarrow \gamma \notin \Gamma\left(\Sigma_{M}, \theta_{M}\right)
$$

- The concurrent alphabet is in bijection with the configurations. It is written as

$$
\Sigma_{M}=\left\{x_{c(t)} \mid c(t) \text { is a configuration }\right\}
$$

All the letters can be easily constructed in parallel assigning a different processor to every letter. 


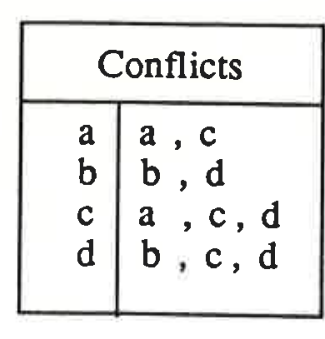

$w=b d a c b a$

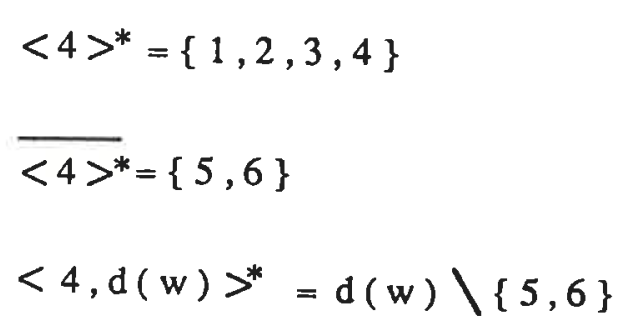

$$
\begin{aligned}
& \Delta^{*}(2)=\{2,4,5,6\} \\
& \overline{\Delta^{*}(2)}=\{1,3\} \\
& \Delta^{*}(2, d(w))=d(w) \backslash\{1,3\}
\end{aligned}
$$
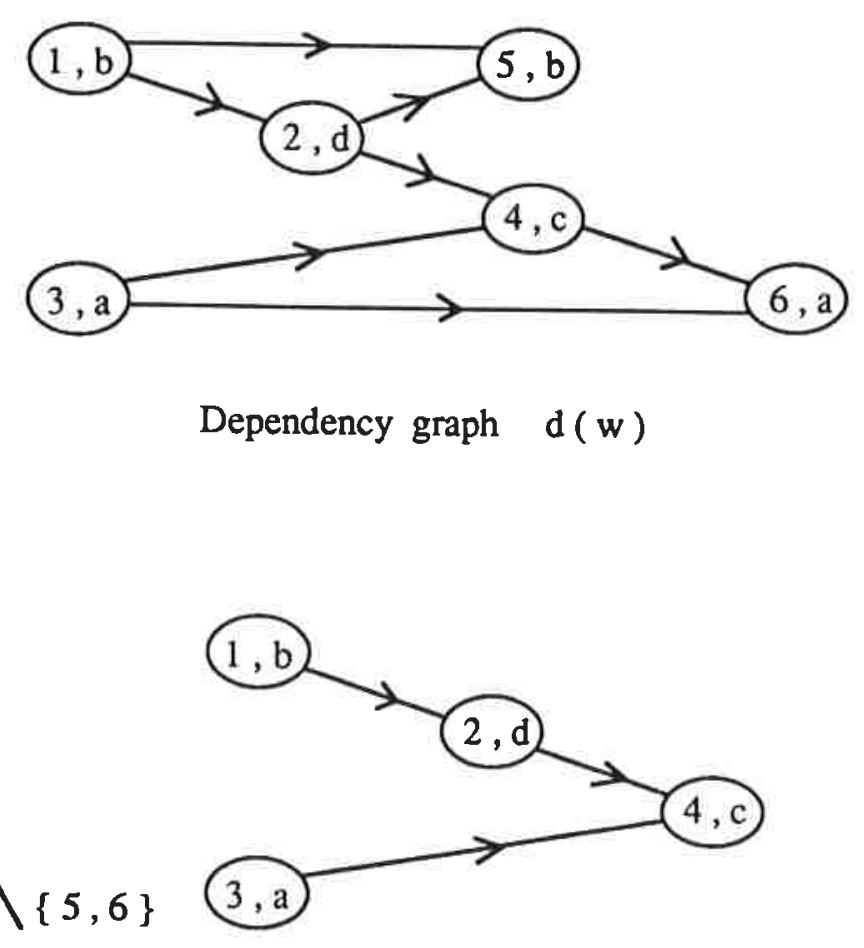

Dependency graph $d(w)$

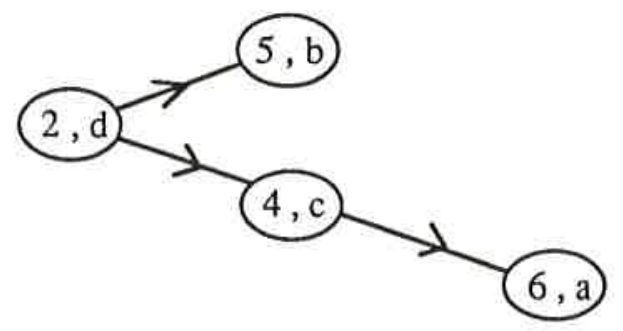

Figure 1. Basic operations in $\Gamma(\Sigma, \theta)$.

- The conflict relation $\overline{\theta_{M}}$ can also be easily constructed in parallel. To do this we assign a processor to a couple of letters.

$$
\begin{aligned}
\overline{\theta_{M}}= & \left\{\left(x_{c(t)}, x_{c(t)}\right) \mid c(t) \text { is a configuration }\right\} \\
& \cup\left\{\left(x_{c(t)}, x_{c(t+1)}\right) \mid c(t) \vdash c(t+1)\right\} \cup\left\{\left(x_{c(t+1)}, x_{c(t)}\right) \mid c(t) \vdash c(t+1)\right\} \\
& \cup\left\{\left(x_{c_{\rho}\left(n^{k}\right)}, x_{c_{i}(0)}\right),\left(x_{c_{i}(0)}, x_{c_{f}\left(n^{k}\right)}\right)\right\} .
\end{aligned}
$$




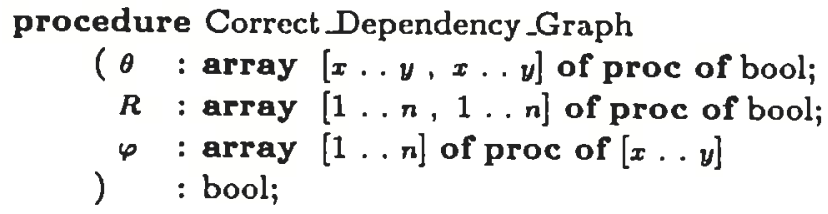

from $N L$ import Transitive_Closure

$(M$ : array $[1 \ldots n, 1 \ldots n]$ of proc of bool

) : array $[1 \ldots n, 1 \ldots n]$ of proc of bool;

$\{$ returns the transitive closure of $M$ \}

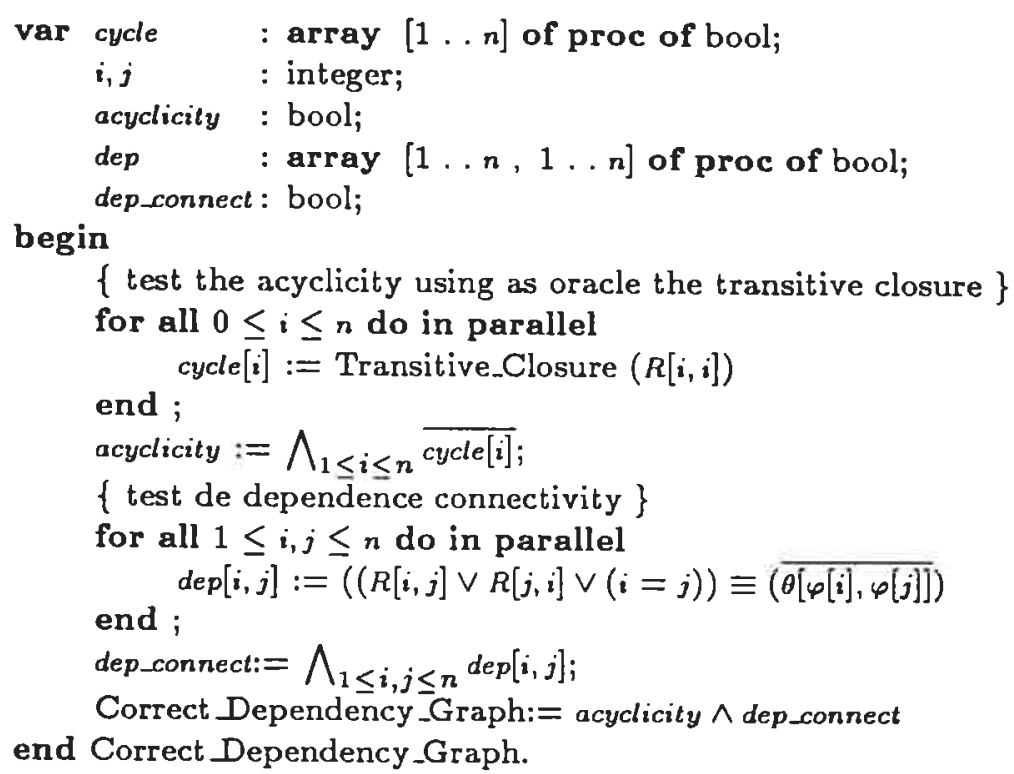

Figure 2. Testing correctness on dependency graphs.

When this relation is constructed we construct $\theta=\Sigma_{M} \times \Sigma_{M} \backslash \overline{\theta_{M}}$ in parallel.

- The dependency graph $\gamma_{M}=\left(V_{M}, R_{M I}, \varphi_{M I}\right)$ is

$$
\begin{aligned}
& V_{M}=\left\{v_{c(t)} \mid c(t) \text { is a configuration }\right\} . \\
& R_{M}=\left\{\left(v_{c(t)}, v_{c(t+1)}\right) \mid c(t) \vdash c(t+1)\right\} \cup\left\{\left(v_{c_{f}\left(n^{k}\right)}, v_{c_{i}(0)}\right)\right\} . \\
& \varphi_{M}\left(v_{c(t)}\right)=x_{c(t)} .
\end{aligned}
$$

When $c_{i}(0) \stackrel{*}{\vdash} c_{f}\left(n^{k}\right)$ we have $\Delta^{*}\left(c_{i}(0)\right) \cap\left\langle c_{f}\left(n^{k}\right)>^{*} \neq \emptyset\right.$ and $R_{M}$ has the following cycle and $\gamma_{M}$ cannot be a tependency graph.

$$
\left(v_{c_{i}(0)}, \ldots, v_{c_{f}\left(n^{k}\right)}, v_{c_{i}(0)}\right)
$$


Reciprocally $c_{f}\left(n^{k}\right)$ does not follows from $c_{i}(0)$ the graph $R$ does not have any cycle and

$$
\begin{aligned}
& R_{M} \cup R_{M}^{-1} \cup\left\{\left(v_{c(t)}, v_{c(t)}\right) \mid v_{c(t)} \in V_{M}\right\}= \\
& \left\{\left(v_{c(t)}, v_{c(t+1)}\right),\left(v_{c(t)}, v_{c(t+1)}\right) \mid c(t) \vdash c(t+1)\right\} \\
& \cup\left\{\left(v_{c_{f}\left(n^{k}\right)}, v_{c_{i}(0)}\right),\left(v_{c_{f}\left(n^{k}\right)}, v_{c_{i}(0)}\right)\right\} \cup\left\{\left(v_{c(t)}, v_{c(t)}\right) \mid v_{c(t)} \in V_{M}\right\}= \\
& \left\{\left(v, v^{\prime}\right) \in V_{M} \times V_{M} \mid\left(\varphi_{M}(v), \varphi_{M}\left(v^{\prime}\right)\right) \in \overline{\theta_{M}}\right\}
\end{aligned}
$$

therefore $\gamma_{M}$ is a dependency graph. That is all.

Given a $\operatorname{dag} G$ and a vertex $v$ we call rank $(v)$ the length of longest path arriving to $v$. We call $D A G \_R A N K$ this problem. It is $N C^{1}$ reducible to $k_{-} C O N N E C T I V I T Y$ because:

$$
\operatorname{rank}(v)=\max \left\{\max \left\{k \mid \operatorname{connect}\left(v, v^{\prime}, k\right)\right\} \mid v^{\prime} \in V\right\} .
$$

Considering a Turing machine with a clock working in log space, we can reduce the problem of acceptance to $D A G \_R A N K$. Therefore $D A G \_R A N K$ is $N L^{*}$ complete under $N C^{1}$ reductions.

Problem 3: The equivalence for dependency graphs problem for concurrent alphabets, called DEPENDENCY _ GRAPHS_ISOMORPHISM is the following:

Input: A concurrent alphabet $(\Sigma, \theta)$ and $\gamma=(V, R, \varphi)$ and $\delta=\left(V^{\prime}, R^{\prime}, \varphi^{\prime}\right)$

Output: It is true that $\gamma, \delta \in \Gamma(\Sigma, \theta)$ and $\gamma \simeq \delta$ ?.

Lemma 4: Given $\gamma=(V, R, \varphi)$ and $\delta=\left(V^{\prime}, R^{\prime}, \varphi^{\prime}\right)$ in $\Gamma(\Sigma, \theta)$, the following conditions are equivalent:

- $\gamma \simeq \delta$.

- for all $i$ such that $0 \leq i \leq \max \{\#(\gamma), \#(\delta)\}$ we have

$$
\{\varphi(v) \mid v \in V, \operatorname{rank}(v)=i\}=\left\{\varphi^{\prime}(v) \mid v \in V^{\prime}, \operatorname{rank}(v)=i\right\}
$$

Proof. Let $\gamma=(V, R, \varphi)$ in $\Gamma(\Sigma, \theta)$ then $\operatorname{rank}(v)=\operatorname{rank}\left(v^{\prime}\right)$ implies $\varphi(v) \neq \varphi\left(v^{\prime}\right)$. Therefore the mapping pairing $V \rightarrow V^{\prime}$ defined by

$$
\operatorname{pairing}(v)=\left\{v^{\prime} \in V^{\prime} \mid \operatorname{rank}(v)=\operatorname{rank}\left(v^{\prime}\right) \wedge \varphi(v)=\varphi^{\prime}\left(v^{\prime}\right)\right\}
$$

give the desired isomorphism.

Lemma 5: The problem DEPENDENCY_ GRAPHS_ISOMORPHISM is $N L^{*}$ complete.

Proof. The program given in the figure 3 show us that the above problem belongs to $N L^{*}$. To prove completeness it is enough to see that $\gamma \simeq \gamma$ is equivalent to $\gamma \in \Gamma(\Sigma, \theta)$. That concludes the proof. 


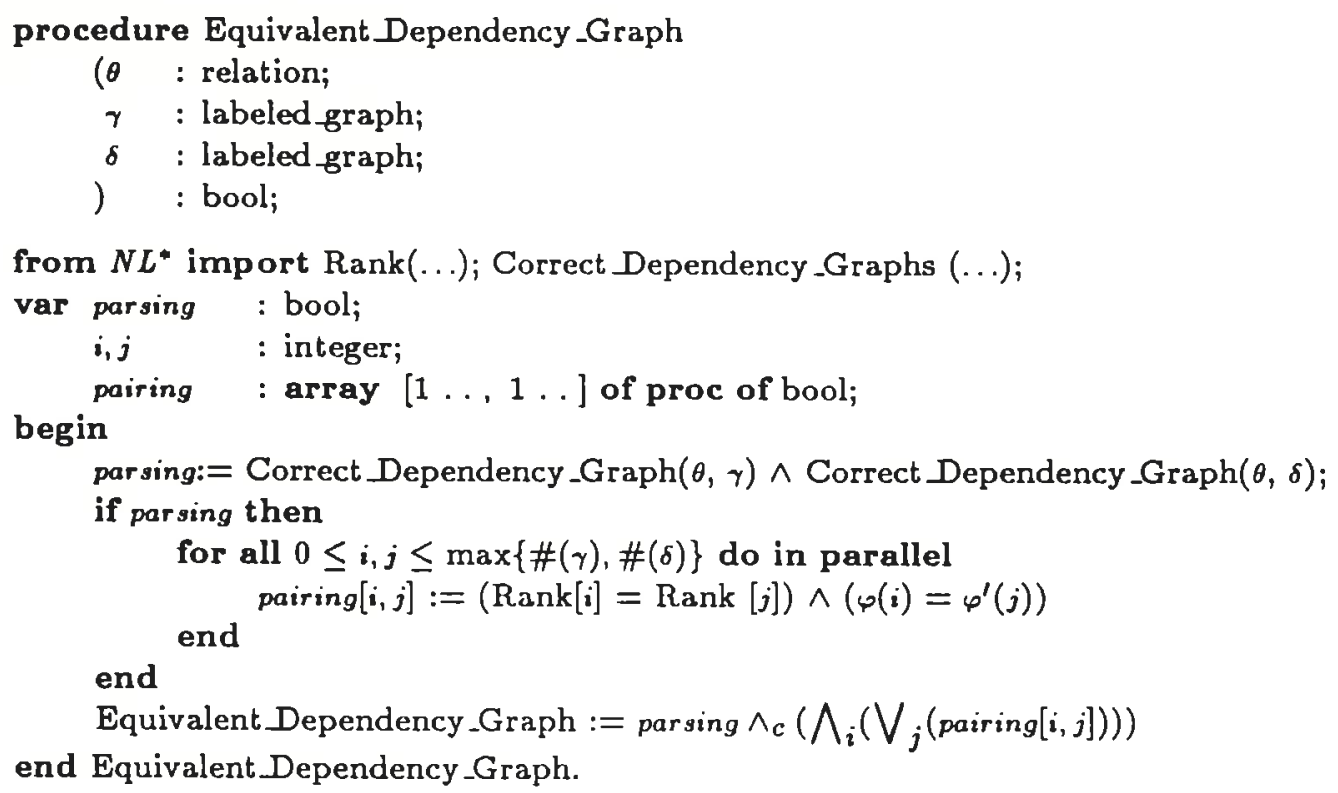

Figure 3. Testing isomorphism on dependency graphs.

Problem 6: Given a dependency graph how to obtain a linearization of it. We call this problem DEPENDENCY _ GRAPHS to_TRACES.

Input: A concurrent alphabet $(\Sigma, \theta)$ and $\gamma \in \Gamma(\Sigma, \theta)$.

Output: A word $w \in \Sigma^{*}$ such that $d(w) \simeq \gamma$.

Given a dependency graph $\gamma=(V, R, \varphi)$ there are many possible linearization (any topological sort is a possible linearization). Between all the solutions we choose the Cartier Foata normal form [CF69], given by the following fact (we write $w \stackrel{*}{\sim} w^{\prime}$ to denote $d(w) \simeq d\left(w^{\prime}\right)$ ) [La79] :

For every word $w \in \Sigma^{+}$exists a unique factorization $w \stackrel{*}{\sim} w_{0} w_{2} \ldots w_{k}$ such that:

(1) For every $0 \leq i \leq k$ the word $w_{i}$ is a monomial of degree 1 on each of its letters (for every $\left.x \in \Sigma,\left|w_{i}\right|_{x} \leq 1\right)$ and any two letters of $w_{i}$ commute.

(2) For every $0 \leq i<k$, each letter of $w_{i+1}$ is in conflict with some letter of $w_{i}$. That means each letter of $w_{i+1}$ either coincides or does not commute with some letter of $w_{i}$.

We write Cartier_Foata $(w)=w_{0} w_{2} \ldots w_{k}$. Given $\gamma=(V, R, \varphi)$ with $k=\max \{r a n k(v) \mid$ $v \in V\}$ and $w \in \Sigma^{+}$such that $\gamma \simeq d(w)$ we have [AR88]

$$
\forall 0 \leq i \leq k: w_{i}=\{\varphi(v) \mid v \in V \wedge \operatorname{rank}(v)=i\}
$$

therefore

$$
\text { Cartier_Foata }(w)=\{\varphi(v) \mid v \in V \wedge \operatorname{rank}(v)=0\} \cdots\{\varphi(v) \mid v \in V \wedge \operatorname{rank}(v)=k\}
$$




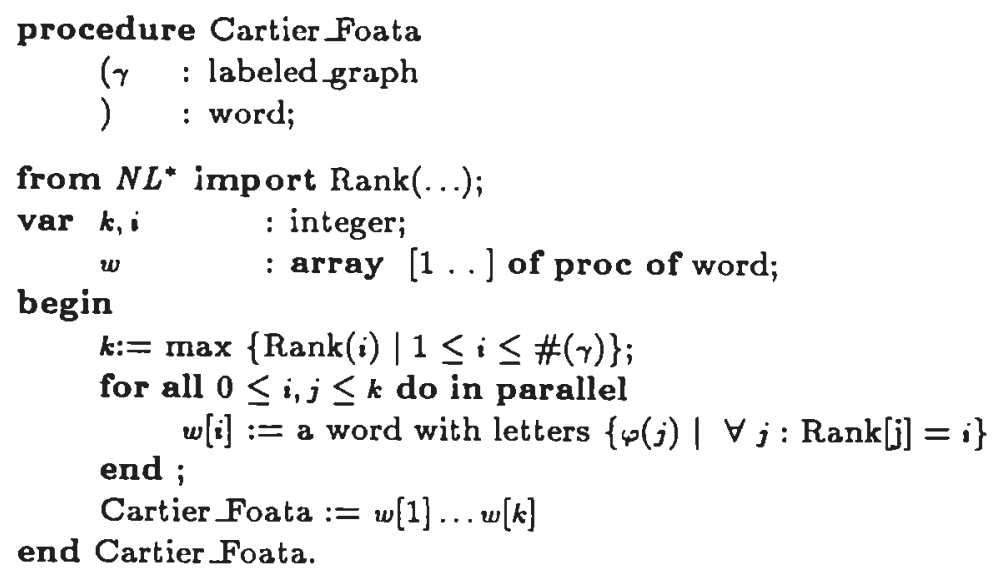

Figure 4. Obtaining the Cartier Foata normal form.

This characterization can be worked out to obtain a parallel algorithm, figure 4 and we get the following result:

Lemma 7: The problem DEPENDENCY_GRAPHS to_TRACES belongs to $N L^{*}$.

Problem 8: The problem TRACES_to_DEPENDENCY_GRAPHS, taking a word and outputting the dependency graph is:

Input: A concurrent alphabet $(\Sigma, \theta)$ and $w \in \Sigma^{*}$.

Output: The dependency graph $d(w)$.

Lemma 9: The problem TRACES_to_DEPENDENCY_GRAPHS belongs to $A C^{0}$.

Proof. All the sentences given in the figure 5 can be coded with operations like "count" or "enumerate". These two operations belongs to $A C^{0}$. That is all.

The precedent lemmas give us an algorithm in $N L^{*}$ to test trace equivalence $w \stackrel{*}{\sim} w^{\prime}$. However it is possible to find a better one belonging to $T C^{0}$ to solve this problem [AG90]. Free partially commutative groups and reduction systems

Given a finite alphabet $\Sigma=\{x, y, z, \ldots\}$ we take a copy $\bar{\Sigma}=\{\bar{x}, \bar{y}, \bar{z}, \ldots\}$ and we write

$$
\hat{\Sigma}=\Sigma \cup \bar{\Sigma}
$$

Given an element $z \in \hat{\Sigma}$ we write $\bar{z}$ as usual. That means if $z=x \in \Sigma$ then $\bar{z}=\bar{x} \in \bar{\Sigma}$, but $z=\bar{x} \in \bar{\Sigma}$ then $\bar{z}=x \in \Sigma$. Given a concurrent alphabet $(\Sigma, \theta)$ we extend the commutation relation to deal with the elements of $\bar{\Sigma}$ :

$$
\hat{\theta}=\theta \cup\{(\bar{x}, y),(x, \bar{y}),(\bar{x}, \bar{y}) \mid(x, y) \in \theta\}
$$




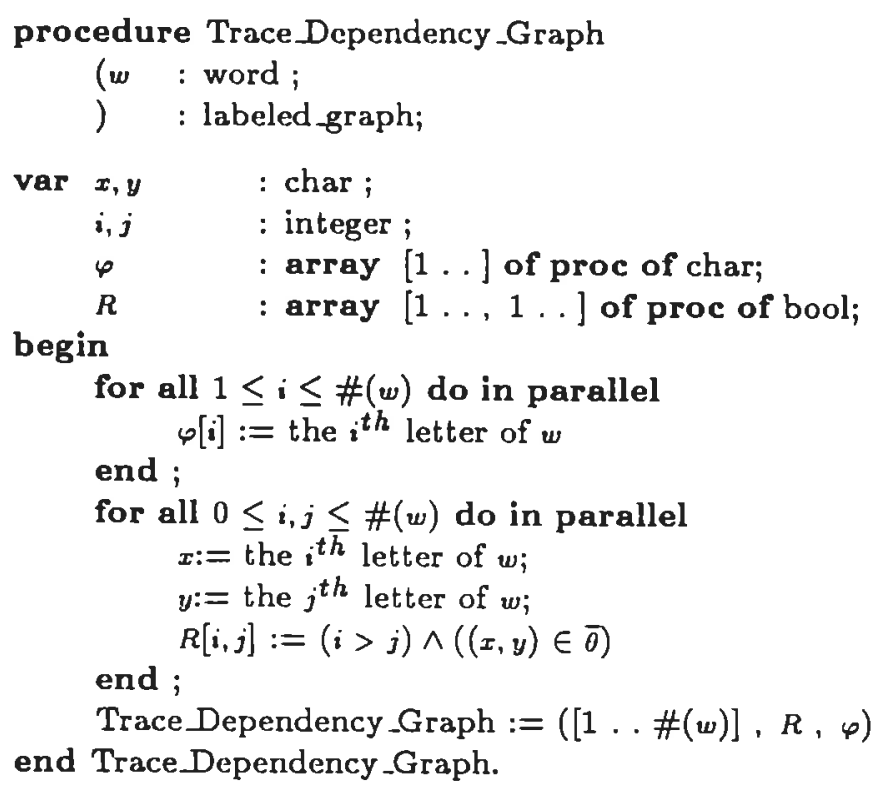

Figure 5. Constructing a dependency graph from a trace.

and we consider the concurrent alphabet $(\hat{\Sigma}, \hat{\theta})$, introduced in [Wr88]. The set of dependency graphs is written $\Gamma(\hat{\Sigma}, \hat{\theta})$. Now we transform $\Gamma(\hat{\Sigma}, \hat{\theta})$ in a reduction system. $(\Gamma(\hat{\Sigma}, \hat{\theta}), \Rightarrow)$. See $[\mathrm{Ja} 88]$ for basic definitions on reduction systems.

Definition 10: Given $\gamma, \delta \in \Gamma(\hat{\Sigma}, \hat{\theta})$ such that $\gamma=(V, R, \varphi)$, we write $\gamma \Rightarrow \delta$ when there are $v, v^{\prime} \in V$ and $z \in \hat{\Sigma}$ such that:

- $\varphi(v)=z, \varphi\left(v^{\prime}\right)=\bar{z}$.

- The longest directed path connecting $v$ and $v^{\prime}$ has length 1 .

- $\gamma \backslash\left\{v, v^{\prime}\right\} \simeq \delta$.

The figure 6 give us an example of reduction.

Let us consider some basic properties of $(\Gamma(\hat{\Sigma}, \hat{\theta}), \Rightarrow)$.

Lemma 11: When $\mu \Leftarrow \gamma \Rightarrow \delta$ then or $\mu \simeq \delta$ or exists $\gamma^{\prime}$ such that $\mu \Rightarrow \gamma^{\prime} \Leftarrow \delta$.

Given $\gamma$ we write $\operatorname{IR} R(\gamma)$ the set of irreducible. By the above lemma we can prove that $\operatorname{IRR}(\gamma)$ has only one element. We write $\operatorname{IR} R(\Gamma)$ for the set of all irreducible elements in $(\Gamma(\hat{\Sigma}, \hat{\theta}), \Rightarrow)$. 
$\mathbf{w}=d \bar{a} c b \bar{c} \bar{b} c d \bar{a}$

\begin{tabular}{|r|l|}
\hline \multicolumn{2}{|c|}{ Conflicts } \\
\hline$a, \bar{a}$ & $a, \bar{a}, c, \bar{c}$ \\
$b, \bar{b}$ & $b, \bar{b}, d, \bar{d}$ \\
$c, \bar{c}$ & $a, \bar{a}, c, \bar{c}, d, \bar{d}$ \\
$d, \bar{d}$ & $b, \bar{b}, c, \bar{c}, d, \bar{d}$ \\
\hline
\end{tabular}

Dependency graph $d(w)$

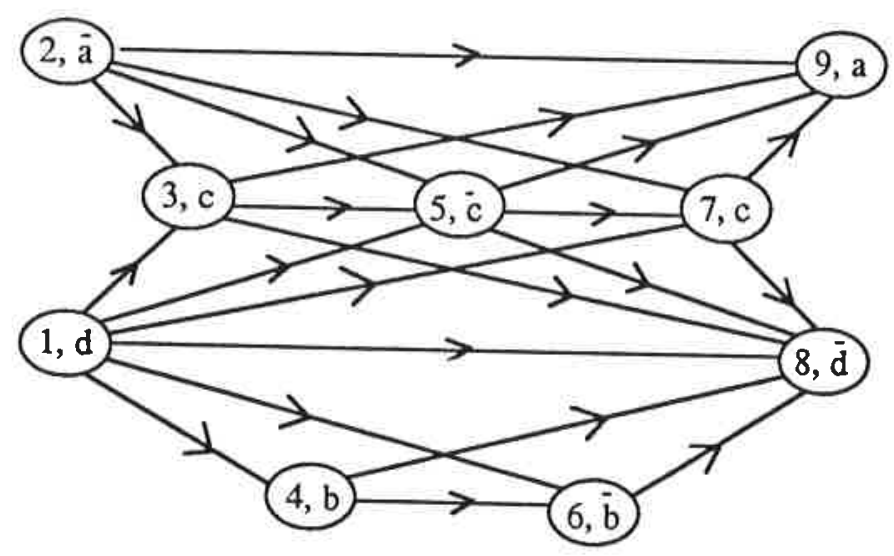

$d(w) \Rightarrow d(w) \backslash\{3,5\}$

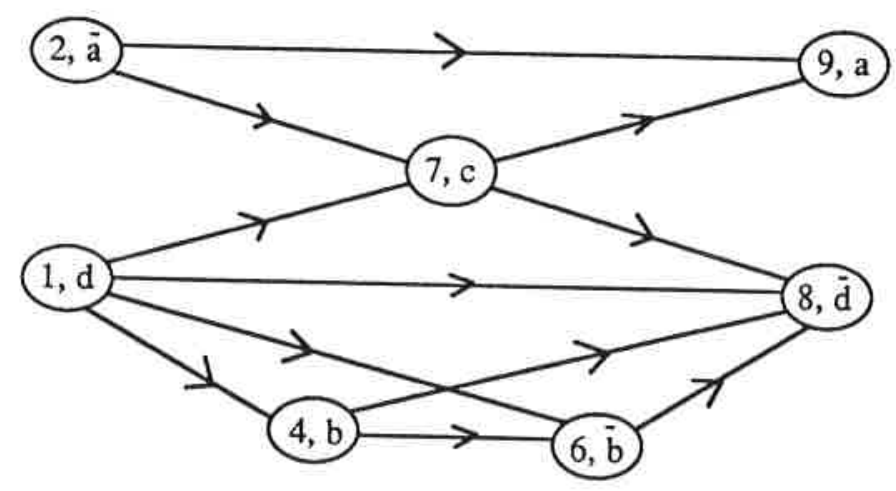

Figure 6. An example of reduction on dependency graphs.

Problem 12: Given a concurrent alphabet an a dependency graph test when it is irreducible. We denote this problem as TEST IRR, it is:

Input: A concurrent alphabet $(\hat{\Sigma}, \hat{\theta})$ and $\gamma=(V, R, \varphi)$.

Output: It is true that $\gamma \in \operatorname{IRR}(\Gamma)$ ?. 
Lemma 13: The problem TESTIRR is $N L^{*}$ complete.

Definition 14: Given $\gamma=(V, R, \varphi)$ we write $\bar{\gamma}=(V, \bar{R}, \bar{\varphi})$, where $\bar{R}=R^{-1}$ and $\bar{\varphi}(z)=\overline{\varphi(z)}$. We call $\bar{\gamma}$ the invers of $\gamma$.

Lemma 15: Given $\gamma, \delta \in I R R(\Gamma)$, the following conditions are equivalent:

- $\gamma \circ \delta \Rightarrow \rho$.

- There exist $z \in \hat{\Sigma}$ and $\gamma^{\prime}, \delta^{\prime} \in I R R(\Gamma)$ such that $\gamma \simeq \gamma^{\prime} \circ z, \delta \simeq \bar{z} \circ \delta^{\prime}$ and $\rho \simeq \gamma^{\prime} \circ \delta^{\prime}$.

Lemma 16: Given $\gamma, \delta \in I R R(\Gamma)$, the following conditions are equivalent:

- $\gamma \circ \delta \stackrel{k}{\Rightarrow} \rho$ and $k \geq 1$.

- There exist $\mu, \gamma^{\prime}, \delta^{\prime} \in I R R(\Gamma)$ such that $\#(\mu)=k$ and $\gamma \simeq \gamma^{\prime} \circ \mu, \delta \simeq \bar{\mu} \circ \delta^{\prime}$ and $\rho \simeq \gamma^{\prime} \circ \delta^{\prime}$.

Lemma 17: The inverses have the following properties:

- $\overline{\gamma \circ \delta}=\bar{\delta} \circ \bar{\gamma}$.

- $\operatorname{IRR}(\gamma \circ \bar{\gamma})=\lambda$.

- $\operatorname{IRR}(\overline{\gamma \circ \delta})=\operatorname{IRR}(\operatorname{IRR}(\bar{\delta}) \circ \operatorname{IRR}(\bar{\gamma}))$.

Given $\gamma$ and $\delta$ we define $\gamma \star \delta=\operatorname{IRR}(\gamma \circ \delta)$. Then $\left(\operatorname{IRR}\left(\Gamma_{\dot{\Sigma}}\right), \star\right)$ is a group isomorphic to the free partially commutative group $G(\theta)$ introduced in [Wr88].

Lemma 18: Given $\gamma, \delta \in \operatorname{IRR}(\Gamma)$, there exists a unique $\mu \in \operatorname{IRR}(\Gamma)$ such that $\gamma \simeq \gamma^{\prime} \circ \mu$ ,$\delta \simeq \bar{\mu} \circ \delta^{\prime}$ and $\operatorname{IRR}(\gamma \circ \delta) \simeq \gamma^{\prime} \circ \delta^{\prime}$.

We call this unique $\mu$ the maximum reducible factor of $\gamma$ and $\delta$ and we write $\mu=$ $M A X \_E D(\gamma, \delta)$.

Lemma 19: Given $\gamma, \delta \in \operatorname{IR} R(\Gamma)$ such that $\gamma=\left(V_{1}, R_{1}, \varphi_{1}\right)$ and $\delta=\left(V_{2}, R_{2}, \varphi_{2}\right)$ we write

$$
\begin{aligned}
& U_{1}=\left\{v \in V_{1} \mid \exists v^{\prime} \in V_{2}: \Delta^{*}(v, \gamma) \simeq \overline{\left\langle v^{\prime}, \delta\right\rangle^{*}}\right\} \\
& U_{2}=\left\{v \in V_{2} \mid \exists v^{\prime} \in V_{1}: \Delta^{*}\left(v^{\prime}, \gamma\right) \simeq \overline{\langle v, \delta\rangle^{*}}\right\}
\end{aligned}
$$

then $M A X_{-} R E D(\gamma, \delta) \simeq \gamma \backslash \overline{U_{1}} \simeq \delta \backslash \overline{U_{2}}$. 


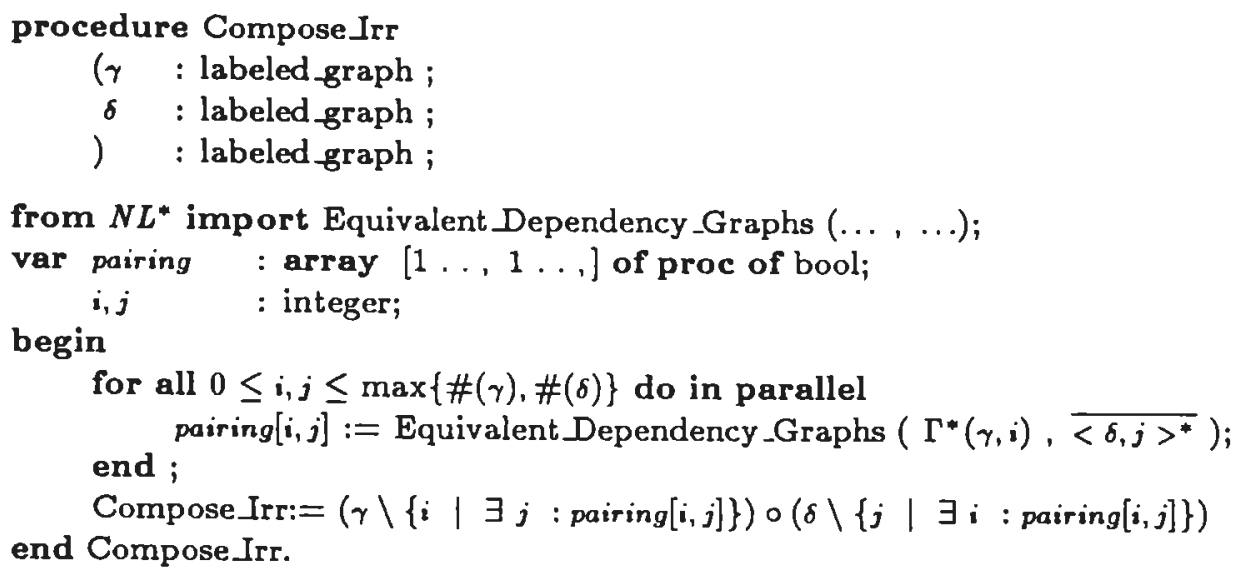

Figure 7. Composing irreducible elements.

Problem 20: Given a concurrent alphabet and two irreducible we define the problem COMPOSEIRR as:

Input: A concurrent alphabet $(\hat{\Sigma}, \hat{\theta})$ and $\gamma, \delta \in I R R(\Gamma)$.

Output: The dependency graph $\operatorname{IR} R(\gamma \circ \delta)$ ?.

Considering the program given in the figure 7 , we have the result given in the following lemma.

Lemma 21: The problem COMPOSE_IRR belongs to $N L^{*}$.

Problem 22: Fixed a concurrent alphabet $(\Sigma, \theta)$ we define FINDIRR as:

Input: Any dependency graph $\gamma \in \Gamma(\Sigma, \theta)$.

Output: The irreducible element $\operatorname{IRR}(\gamma)$.

Considering the algorithm given in the figure 8 we obtain the following lemma

Lemma 23: The problem FIND IRR belongs to $N C$.

Problem 24: We define the word problem for free partially commutative monoids, written WORD_PROBLEM_FPCG as:

Input: A concurrent alphabet $(\Sigma, \theta)$ and two graphs $\gamma=(V, R, \varphi)$ and $\delta=\left(V^{\prime}, R^{\prime}, \varphi^{\prime}\right)$. Output: It is true that $\gamma, \delta \in \Gamma(\Sigma, \theta)$ and $\gamma \stackrel{*}{\Longleftrightarrow} \delta$ ?. 


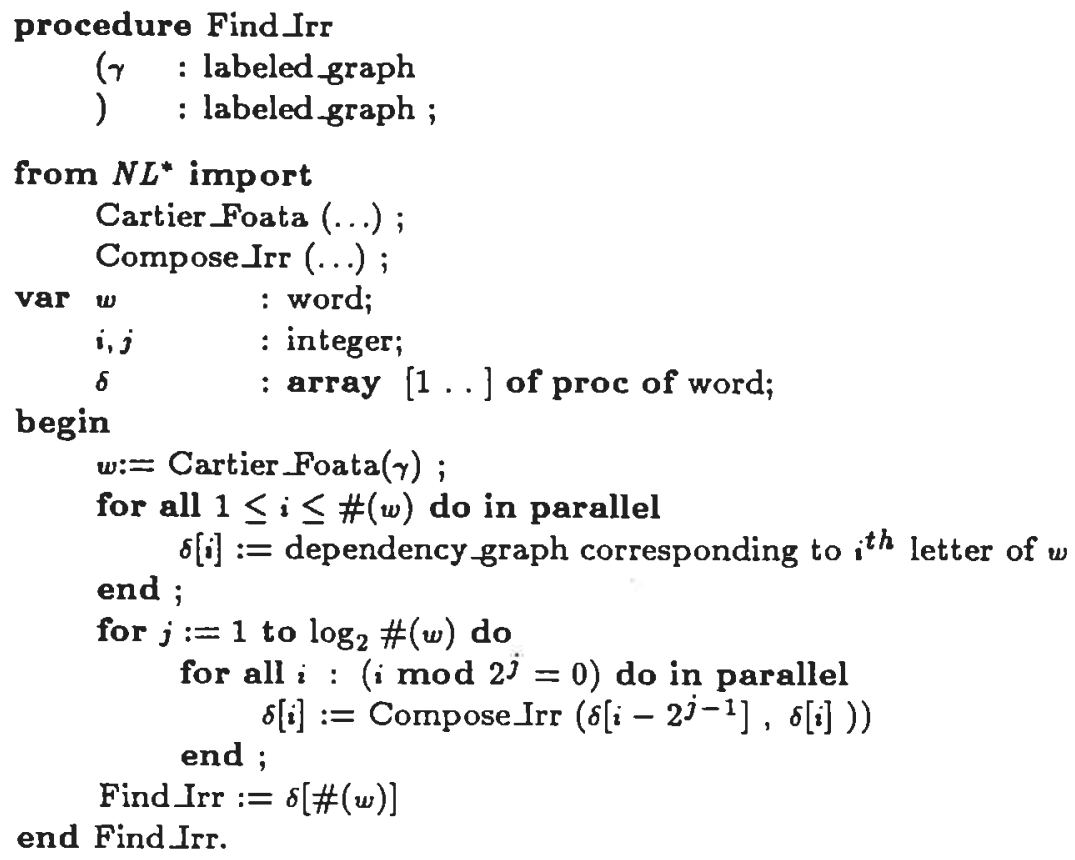

Figure 8. Finding $\operatorname{IRR}(\delta)$.

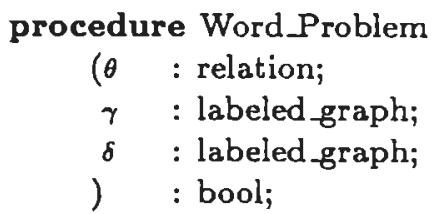

from $N C$ import

Find $\operatorname{Irr}(. .$.$) ;$

Correct Dependency_Graphs (...); begin

Equivalent_Dependency_Graphs (...);

Word_Problem: $=$ Correct_Dependency_Graph $(\theta, \gamma) \wedge$ Correct_Dependency_Graph $(\theta, \delta)$ $\wedge_{c}$

end Word_Problem.

Equivalent_Dependency_Graph (Find $\operatorname{Irr}(\gamma), \operatorname{Find} \operatorname{Irr}(\delta)$ )

Figure 9. Word problem for free partially commutative monoids.

Lemma 25: The problem $W P \_P C G$ belongs to $N C$.

Proof. Consider the algorithm given in the figure 9. 
It is also possible to represent traces by a set of sincronizet words called histories. This idea has been developed by A. Mazurkiewicz in [Ma88]. There exists a linear sequential time algorithm [Wr88] to solve word problem for free partially commutative groups. This algorithm uses histories to represent traces and works with a set of sincronizet puhsdowns.

\section{References}

[ABGS91] Álvarez, C; Balcázar, J.L.; Gabarró, J; Sántha, M.: Parallel complexity in the design and analysis of concurrent systems. PARLE 91, Lecture Notes in Computer Science 505 (1991) 288-303.

[AG91] Álvarez, C; Gabarró, J.: The parallel complexity of two problems on concurrency. Information Processing Letters 38 (1991) 61-70.

[AR88] Aalbersberg, IJ, J; Rozenberg, G.: Theory of traces. Theoretical Computer Science 60 (1988) 1-82.

[BDG88] Balcázar, J.L.; Díaz , J. Gabarró, J: Structural Complexity I. EATCS Monographs on Theoretical Computer Science, 11, Springer-Verlag 1988.

[BDG90] Balcázar, J.L.; Díaz , J. Gabarró, J: Structural Complexity II. EATCS Monographs on Theoretical Computer Science, 22, Springer-Verlag 1990.

[Co85] Cook, S.: A taxonomy of problems with fast parallel algorithms. Information and Control 64 (1985) 2-22.

[CF69] Cartier, P., Foata, D.: Problèmes Combinatoires de Commutations et Réarran gements. Lecture Notes in Mathematics, 85 (1969).

[GG91] Gabarró, J; Gavaldà, R.: An approach to correctness of data parallel algorithms. Report LSI-91-19, Univ. Politècnica de Catalunya.

[HS86] Hillis, D.W.; Steele, G.L.: Data parallel algorithms. Communications of the ACM 29 1170-1183 (1986).

[La79] Lallement, G.: Semigroups and Combinatorial Applications. Wiley-Interscience 1979.

[Ja88] Jantzen, M.: Confluent String Rewriting. EATCS Monographs on Theoretical Computer Science, 14 Springer-Verlag 1988.

[Ma88] Mazurkiewicz, A.: Basic Notions of Traces, Lecture Notes in Computer Science 354 (1989) 285-363.

[Wr88] Wrathall , C.: The word problem for free partially commutative groups. J. Symbolic Computation 6 (1988) 99-104. 